\title{
Financial impact of covid-19 on ophthalmic practices
}

\author{
Nishant Renu* \\ *Correspondence: nishant.sinha11@gmail.com

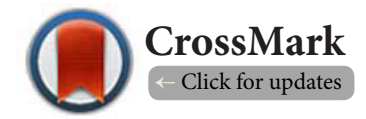

Westcliff University, 17877 Von Karman Ave \#400, Irvine, CA 92614, USA.

\begin{abstract}
The unprecedented threat of Covid-19 has forced transition from the normal way of life. The new norm now includes social distancing, the use of face masks, and reduced contact to only necessary cases. In ophthalmology, practice procedures are currently only limited to emergency procedures such as trauma and endophthalmitis. The state of affairs, as described in this review, is greatly reduced the number of patients seeking ophthalmology services. There are fewer patients making visits as a result of extreme caution on both their parts and the medics. Expectedly, many private practitioners are feeling the financial pinch, with as many as $90 \%$ of private practices seeking federal loans, among other initiatives to cushion their practices. Despite these efforts, the outlook is grim, with an estimated $6 \%$ of private practices expected to close, while those that pull through are predicted to be smaller in size and poor financial positions.
\end{abstract}

Keywords: Ophthalmology practice, COVID-19, financial position

\section{Introduction}

The global economy is at a near standstill because of the effects of the Covid-19 pandemic. Internationally and nationally, businesses are coming to terms with broken supply chains, reduced consumer spending, and liquidity challenges [1]. All these challenges transcend professions equally affecting business across the country, including ophthalmology practices. As such, it is of importance to study the effects of the current economic downturn from an industry-based point of view to appreciate how industry-unique factors influence the impact of the tough economic times and how business is responding. For this review, I referred to all the latest news updates, articles, websites, interviews, and peer-reviewed articles pertaining to Covid-19 and used Google Scholar and Scopus for the literature search to evaluates the financial impact of Covid-19 on ophthalmic practice in the United States. To this end, it considers the effects the diseases and preventative measures have affected patient volumes and the availability of procedures. The paper then proceeds to contextualize the preceding section by elaborating on the financial position of the industry generally. Thirdly and finally, the paper evaluates the impact of government initiatives designed to ameliorate the impact of the disease.

\section{Review}

Practice with Limited Physical Contact

The unprecedented threat of Covid-19 has forced a transition from the normal way of life. The new norm now includes social distancing, the use of face masks, and reduced contact to only necessary cases. In the medical field, a duty to protect patients and the community at large forced a spontaneous evolution of the normal practice. Like many other close contact human activities, medical procedures and practices are now tempered with preventive measures intentionally designed to limit the spread of the disease. This state of affairs demands that practitioners reevaluate traditional physical examination as part of patient management in both private and public practices [2]. Since most of the private practices are owned by doctors or groups of doctors, and it is becoming a daunting place without an influx of patients. In lieu of cash flow, it is getting difficult for them to manage their practice, pay their nurse and clinical staff, their salaries are being frozen or reduced, and some are being furloughed [3]. On the contrary, public practices owned by hospitals or academic centers are flooded with patients suffering from Covid-19 and trying to provide patients optimum care in this critical situation with their limited resources [17].

The risk of contributing to the spread of the Covid- 19 by both patients and medical staff has necessitated increased reliance on alternatives to physical patient visits. As such, doctors are limited to consultations via telephones or telemedicine. Expectedly, telemedicine has gained popularity in the wake of the pandemic. These new forms of offering medical services build on systems and structures that have matured since its 
Nishant Renu, Journal of Eye and Ophthalmology 2021,

http://www.hoajonline.com/journals/pdf/2055-2408-8-1.pdf

doi: $10.7243 / 2055-2408-8-1$

inception in the early 1990s [18]. With this tool, patients can get the medical services and prescriptions they need without the need for direct face-to-face contact with a physician using their phone, laptop, or computer. However, these services are only limited to only a few outpatient services where a minimal diagnosis is required. Several companies are currently offering practices with a telehealth and telemedicine platform at no cost to facilitate telehealth and telemedicine adoption. It is anticipated that there will be a surge in the acceptance rate of these telemedicine platforms among both patients and health care providers [4]. These strategies are the result of medics implementing recommendations on how to prevent the spread of the diseases and protect the larger community from the high virulence of the disease [2]. However, the safety procedures are a trade-off as most medical procedures are currently only limited to emergency procedures such as trauma and endophthalmitis. As such, the number of patient visits has dramatically decreased across all medical specialties, including ophthalmology practices [5]. Statistics during the early phase of the pandemic showed that ophthalmology practices experienced a $60 \%$ dip in client visits [6]. In other surveys, changes in visit volume specific to ophthalmology were a staggering $75 \%$ [7]. All ophthalmology specialties, such as cataract and refractive, oculoplastic, retina, glaucoma, etc., with their main focus primarily on outpatient care, have shifted dramatically, focusing on an emergency or emergent treatment to ensure the wellbeing of all patients and health staff and to conserve surgical equipment and resources. However, in recent months, ambulatory services are witnessing increased patient foot traffic, though the numbers are still about one-third of the normal figures. According to Mehrotra et al. [6]., ophthalmology practices experienced the highest decrease in-patient visits but, in recent times, also experienced among the highest improvement in terms of patient visits. Similarly, we can see the rebound in visits occurring across all specialties. The relative decline in visits remains the largest among surgical, pediatrics, and procedural specialties. The relative decline is of a lower degree in other specialties such as behavioral health, rheumatology, oncology, gynecology, endocrinology, and adult primary care [6].

Ophthalmology practices are highly susceptible to spreading the disease because of the nature of the practice. This is because the practice relies on reusable equipment and close human contact. The extremely close contact required for most ocular surface examinations (often only a few inches), and the limitations of using equipment to perform eye testing with protective gear, make this area of medicine even more high-risk for patient-physician transmission than others [8]. In addition, there is understandable skepticism among practitioners on when they or their facilities will resume normal practice, such as elective surgeries. Besides, doctors have to contend with the ethical dilemma of evaluating the level of seriousness of patients' immediate needs for the safety of the patient, associate staff members, and the community who are at risk of infection with increased human to human contact [9]. Consequently, there are fewer patients making visits as a result of restrain on both their parts and the medics [10]. Collectively, stakeholders are managing the situation better at the moment, as evidenced by the increase in the number of patient visits. In essence, the pandemic had brought to a near standstill ophthalmology practices' access to consumers.

\section{Financial Strain in the Industry}

The financial impact of the current state of affairs is farreaching. The most affected business models are those based on consumers. There is consensus among economists that the pandemic will have far-reaching effects both in the short term and medium term. In addition, a majority of businesses will experience challenges associated with the effects of the restriction on movement and quarantines, with the worst-hit organizations being business based on consumer spending [11]. These predictions do not bode well for the consumerbased ophthalmology practice. The effects of the pandemic manifesting survey on the performance of ophthalmology practice illustrate the magnitude of impact the pandemic has had on private practice. According to the American Academy of Ophthalmology (AAO), without government support, many practitioners risk having a smaller and financially unstable business. To illustrate the magnitude of the bleak state of affairs, AAO's study predicts that $47 \%$ of practices will be smaller and financially unhealthy [12]. Besides, only $6 \%$ are projected to bounce back after the pandemic to patient volumes and financial status to pre-COVID times. Concomitantly, $6 \%$ of practices in the country are expected to close, while a faction of the businesses at $14 \%$ is expected to financially healthy [13]. The precarious financial and operational position medics find themselves in is a result of several factors, including steady overhead costs coupled with reimbursement cuts from both insurers and government payers. Thus, from a business point of the situation looks bleak for ophthalmology practices, particularly those unable to secure government safety nets.

Heavy Reliance by Private Practices on Government Aid In March of 2020, President Trump assented to the 2 billion dollars Coronavirus Aid, Relief, and Economic Security (CARES) Act. The program targets business owners who are facing difficult financial times as a result of the pandemic by offering financial aid [14]. The Act allocates an estimated 376 billion dollars towards helping business owners see through the worst of the economic effects of the pandemic. The mere fact that the government deemed it fit to provide a safety net exemplifies the magnitude of the economic impact the pandemic has had on businesses. Despite access to government aid in various forms, Donnenfeld and Devgan [12] clarify that access to these grants and loans are a challenge for many private ophthalmology practices. According to the AAO, procedural red tape and funding were depleted [13]. Despite these challenges, practitioners are taking advantage 
of the various financial aid programs available. Donnenfeld and Devgan point out that federal loans are the most popular program among practitioners, with $92 \%$ reporting intention to apply for these forms of financial support. The paycheck protection program is the next popular initiative, with an estimated $80 \%$ reported having intending to apply under the program [12]. Hence, in practice, the CARES Act is a welcome relief for many businesses, including ophthalmology, particularly those in private practice.

\section{The Future}

Long term, the practice of ophthalmology has all the signs of bouncing back. Recent surveys on changes in visit volume show marked improvement. According to some quotas number of patients visiting ophthalmologists has significantly improved so much that current numbers are an estimated $30 \%$ lower than pre-Covid-19 [6]. Though the increased patient volumes are nationwide, the best performing areas are in the South Central region. The laws of supply and demand dictate pricing; hence variations on either side of the equation lead to a corresponding alteration in pricing. To play the devil's advocate, the statistics show that the number of practitioners will invariably decline as a result of the economic chokehold businesses are experiencing. As a result, the supply of practices will decline. For those savvy enough to withstand the current storm, then lower ophthalmology practice supply works to their advantage in terms of profitability. This is good news, particularly for operations banking on government loans to bail them out at the current time. Moreover, these assertions are not baseless, as illustrated by statistics of plateauing telemedicine as some semblance of normalcy returns [6]. In the future, the prospect of servicing these loans and overcoming unhealthy financial situations will be good and, therefore, positive news for the practice.

There is, however, cautious optimism considering recent events. Once the curve showed signs of flattening and the public wary of staying indoors, many parts of the country started opening up. Unfortunately, these events invariably lead to an increase in the number of new infections, with current estimates placed at 2 million new cases [15]. These events coincide with President Trump's Administration encouraging medical facilities to reopen [16]. How all these new factors affect business and the medical profession is anyone's guess. This study has potential limitations. The financial impact on Ophthalmology practices demonstrated in this study is only valid for the United States. Further, it doesn't address or quantify the impact of COVID 19 on each specialty in the ophthalmology space. Further research or study needs to be conducted to analyze the effect of Covid-19 on each ophthalmology sub-specialty individually.

\section{Conclusion}

In conclusion, the COVID-19 pandemic has had tremendous and far-reaching consequences for medical practices and businesses alike. Ophthalmology practice, in particular, has been hard because of the consumer-based nature of the business and the high probability of infection within the practice. In the country, many private practitioners are staring at tough economic times, with a significant portion of practitioners risking losing their business or barely holding onto the businesses. The government's stimulus package is a welcome reprieve for many businesses; however, it is not a silver bullet for the unique challenges facing the industry and the collective business environment. Despite the current poor state of ophthalmology practices in the medium to long term, businesses that can weather the current storm have something positive to look forward to. Moreover, the industry so far illustrates its resilience and ability for a majority of businesses to survive the current pandemic.

\section{Competing interests}

The author declares that he has no competing interests.

\section{Acknowledgements}

I am incredibly thankful to my colleague Sean Tighe for his encouragement and support in writing this article.

\section{Publication history}

Editor: Carsten H. Meyer, Pallas Clinic, Switzerland. Received: 07-Dec-2020 Final Revised: 07-Feb-2021 Accepted: 28-Feb-2021 Published: 17-Mar-2021

\section{References}

1. Fernandes N. Economic effects of Coronavirus outbreak (COVID-19) on the world economy. SSRN Electron J [Internet]. 2020; Available from: http://dx.doi.org/10.2139/ssrn.3557504.

2. Ferneini EM. The financial impact of COVID-19 on our practice. J Oral Maxillofac Surg. 2020;78(7):1047-8.

3. Cutler D. How will COVID-19 affect the health care economy? JAMA. 2020;323(22):2237-8. doi:10.1001/jama.2020.7308.

4. Fisk M, Livingstone A, Pit SW. Telehealth in the context of COVID-19: Changing perspectives in Australia, the United Kingdom, and the United States (preprint) [Internet]. JMIR Preprints. 2020. Available from: http:// dx.doi.org/10.2196/preprints.19264.

5. Lim LW, Yip LW, Tay HW, Ang XL, Lee LK, Chin CF, et al. Sustainable practice of ophthalmology during COVID-19: challenges and solutions. Arbeitsphysiologie. 2020;258(7):1427-36.

6. Mehrotra A, Chernew M, Linetsky D, Hatch H, Cutler D. What impact has COVID-19 had on outpatient visits? [Internet]. Commonwealth Fund; 2020 [cited 2020 May 25]. Available from: http://dx.doi.org/10.26099/ DS9E-JM36

7. Wong TY, Bandello F. Academic Ophthalmology during and after the COVID-19 Pandemic. Ophthalmology. 2020;127(8):e51-2.

8. Robbins SL, Packwood EA, Siegel LM, AAPOS Socioeconomic Committee. Economic impact on pediatric ophthalmologists in the time of COVID-19. J AAPOS [Internet]. 2020; Available from: http://dx.doi.org/10.1016/j. jaapos.2020.06.002

9. Chan W-M, Liu DTL, Chan PKS, Chong KKL, Yuen KSC, Chiu TYH, et al. Precautions in ophthalmic practice in a hospital with a major acute SARS outbreak: an experience from Hong Kong. EYE. 2006;20(3):283-9.

10. Lai THT, Tang EWH, Chau SKY, Fung KSC, Li KKW. Stepping up infection control measures in ophthalmology during the novel coronavirus outbreak: an experience from Hong Kong. Arbeitsphysiologie. 2020;258(5):1049-55.

11. Craven M, Liu L, Mysore M, Wilson M. COVID-19: Implications for 
Nishant Renu, Journal of Eye and Ophthalmology 2021,

http://www.hoajonline.com/journals/pdf/2055-2408-8-1.pdf

business. McKinsey \& Company. 2020.

12. Donnenfeld $E$ and Devgan U. AAO COVID-19 survey shows revenue losses for private practices [Internet]. Healio.com. 2020 [cited 2020 May 25]. Available from: https://www.healio.com/news/ ophthalmology/20200521/aao-covid19-survey-shows-revenue-lossesfor-private-practices

13. AAO survey reveals pandemic's effect on private ophthalmic practices [Internet]. Eyewire.news. 2020 [cited 2020 May 25]. Available from: https://eyewire.news/articles/aao-survey-reveals-pandemics-dire-effecton-private-ophthalmic-practices/

14. Mountain TP. Coronavirus aid, relief, and economic security act (CARES) tax rebates [Internet]. Lib.vt.edu. 2020 [cited 2020 May 25]. Available from: https://vtechworks.lib.vt.edu/bitstream/handle/10919/98289/ AAEC-219.pdf?sequence $=1$

15. Chappell B. U.S. hits 2 million Coronavirus cases as many states see A surge of patients. NPR [Internet]. 2020 Jun 10 [cited 2020 Jun 12]; Available from: https://www.npr.org/sections/coronavirus-liveupdates/2020/06/10/873473805/u-s-hits-2-million-coronavirus-cases-asmany-states-see-a-surge-of-patients

16. Reed T. Trump administration pushes for healthcare facilities to reopen [Internet]. Fiercehealthcare.com. 2020 [cited 2020 Jun 12]. Available from: https://www.fiercehealthcare.com/hospitals/trumpadministration-releases-reopening-guide-for-healthcare-facilities

17. Starr MR, Israilevich R, Zhitnitsky M, Cheng QE, Soares RR, Patel LG, et al. Practice patterns and responsiveness to simulated common ocular complaints among US ophthalmology centers during the COVID-19 pandemic. JAMA Ophthalmol [Internet]. 2020; Available from: http:// dx.doi.org/10.1001/jamaophthalmol.2020.3237

18. Mahar JH, Rosencrance JG, Rasmussen PA. Telemedicine: Past, present, and future. Cleve Clin J Med. 2018;85(12):938-42.

\section{Citation:}

Renu N. Financial impact of covid-19 on ophthalmic practices. J Eye Ophthalmol. 2021; 8:1.

http://dx.doi.org/10.7243/2055-2408-8-1 